\title{
Visita de abejas (Apis mellifera, Hymenoptera: Apoidea) a flores de melón Cucumis melo (Cucurvitaceae) en Panamá
}

\author{
Juan Carlos Di Trani de la Hoz \\ Apdo. 0830-00440, San Francisco, Panamá, Panamá; juanditrani@yahoo.com
}

Recibido 02-XII-2003. Corregido 11-I-2007. Aceptado 15-I-2007.

\begin{abstract}
Bee(Apis mellifera, Hymenoptera: Apoidea) visitation to cantaloupe Cucumis melo (Cucurvitaceae) flowers in Panama. Flower visits by bees were observed in a melon cultivated field of San Lorenzo district, in Chiriquí, Panama, from January 6 to February 19, 2002, from 6:30 am to 4:30 pm We recorded the duration of each foraging event and the type of resource collected. Flower visits were mostly for nectar collection ( $75 \%)$. Pollen foraging was concentrated in the first hours of the morning and ended by 11:00 am The mean collection time was similar for both food resources, but was different between flower sexes. Flower visits to female flowers took longer (Student's t test, $\mathrm{p}<0.0001$ ), with a mean time duration of $8.4 \pm 4.4 \mathrm{~s}$, whereas in male flowers mean visitation time was of $4.0 \pm 1.5 \mathrm{~s}$. Finally, the mean time for each floral sex remained practically constant through the evolution of the crop. Our results were similar to the found ones in temperate zone crops, so apparently tropical conditions of Panama do not change the bee visit patterns on melon flowers. Rev. Biol. Trop. 55 (2): 677-680. Epub 2007 June, 29.
\end{abstract}

Key words: melon, pollination, flower visits, resource, nectar, pollen, flower sex.

La planta del melón es de tipo andromonoico, esto quiere decir que produce flores estaminadas y hermafroditas. Las flores masculinas se empiezan a producir una o dos semanas antes que las femeninas (McGregor 1976), y son mucho más abundantes. Las flores del melón permanecen abiertas un solo día. Abren inmediatamente con la salida del sol, o un par de horas después, aunque bajas temperaturas, alta humedad o nubosidad suelen retrasar el suceso (McGregor y Todd 1952).

Se trata de una planta autocompatible y aunque el ovario es receptivo al polen de la misma flor, se obtienen considerables beneficios cuando el polen proviene de otra flor (polinización cruzada), ya que es más factible que lleguen al estigma los granos de polen de la variedad adecuada, en el momento de mayor receptibilidad, y en mayor cantidad (Free 1970, McGregor 1976, Rallo García, 1986).
En el melón, la polinización es realizada casi exclusivamente por abejas, particularmente las melíferas (McGregor y Todd 1952, Taylor 1955, McGregor et al. 1965, Iselin et al. 1974, Gary et al. 1975). Los beneficios de la polinización con abejas incluyen mayor rendimiento y mejor calidad de las cosechas (McGregor 1976).

Debido a que en las cucurbitáceas los granos de polen producen túbulos de polen directamente hacia abajo, y con muy poco movimiento lateral, una cantidad insuficiente de granos de polen en uno de los lóbulos producirá eventualmente frutos asimétricos o enanos (Adlerz 1966). En el melón, la producción de frutos de forma comercial (grandes y simétricos) requiere que se depositen al menos 400 granos de polen sobre los tres lóbulos del estigma (Gary et al. 1975). Para ello, algunos estudios como el de McGregor y Todd (1952) 
han demostrado que se requieren al menos diez visitas de abejas por flor. Sin embargo, casi todos estos estudios han sido realizados en climas templados y tomando en cuenta sólo a las abejas melíferas. Por ello, resulta importante conocer algunas de las características de las visitas de las abejas a las flores de campos en el trópico. Entre ellas, el tiempo medio de visita, la preferencia por los recursos visitados, el tiempo empleado en cada sexo, y el horario de visita, para obtener un mejor rendimiento en la polinización. En este informe se analizan los patrones de visitas y el comportamiento de abejas melíferas, Apis mellifera, en Panamá.

\section{MATERIALES Y MÉTODOS}

Entre el 6 de enero y el 19 de febrero del 2002 se registraron las visitas y el comportamiento de las abejas en flores de melón de un campo del distrito de San Lorenzo, Chiriquí, Panamá. Para ello se seleccionó una flor control masculina y una femenina por día y se anotaron las visitas que recibían desde las 6:30 am hasta las 4:30 pm, en intervalos de una hora, con recesos de media hora entre ellos (Cuadro 1). Sólo se contabilizaron las "visitas efectivas", es decir, cuando la abeja permaneció en la flor por más de $2 \mathrm{~s}$, tiempo mínimo estimado para que la abeja entrara en contacto con las partes sexuales de la flor. El tipo de recurso visitado se determinó indirectamente, por la presencia de polen en las corbículas de la abeja. Finalmente se aplicó el análisis estadístico t de Student para determinar si las diferencias en el tiempo de visitas fueron significativas. Apis mellifera.

El material testigo está en la colección de la Maestría en Entomología de la Universidad de Panamá, Panamá.

\section{RESULTADOS}

Elección de recursos: Durante el período de muestreo, la mayor parte de las visitas observadas fueron dedicadas a la recolección
CUADRO 1

Horario de visitas por recursos del 6 enero a 19 febrero 2002

TABLE 1

Visits schedule per resources from January 6 to February 19, 2002

$\begin{array}{lccc}\begin{array}{l}\text { Período de } \\ \text { observación }\end{array} & \text { Néctar } & \text { Polen } & \text { Total x Periodo } \\ \text { 6:30-7:30 } & 36 & 53 & 89 \\ \text { 8:00-9:00 } & 30 & 179 & 209 \\ \text { 9:30-10:30 } & 175 & 142 & 317 \\ \text { 11:00-12:00 } & 179 & 0 & 179 \\ \text { 12:30-1:30 } & 241 & 0 & 241 \\ \text { 2:00-3:00 } & 111 & 0 & 111 \\ \text { 3:30-4:30 } & 104 & 0 & 104 \\ \text { Total de visitas } & 876 & 374 & 1250\end{array}$

de néctar (878 visitas). Para la recolección de polen, por otro lado fueron dedicadas menos de la mitad de las visitas, tan sólo 333 (Fig. 1).

Horario de visita: La actividad colectora diaria de las abejas se inició poco después de las seis de la mañana, con pocas visitas, y se fue incrementando en los períodos siguientes. Para el período de 9:30 a 10:30 am, se alcanzó

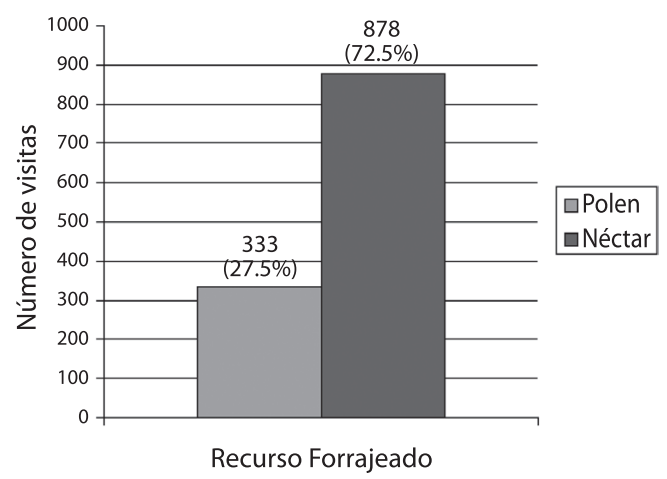

Fig.1. Proporción de visitas por recurso, flores "control" del 6 enero al 19 de febrero 2002.

Fig.1. Visits proportion per floral resource on control flowers from January 6 to February 19, 2002. 
el clímax de visitas, donde se contabilizaron algo más de un cuarto de las visitas diarias, casi todas para la recolección de polen. Luego, en el período de 11:00 am a 12:00 md, se experimentó un descenso en la actividad, seguido de un repunte en el siguiente período, de 12:30 a 1:30 pm. A partir de ese período, la actividad declina, hasta ser casi nula, llegando a las $5 \mathrm{pm}$. De tal modo, la mayor parte de las visitas, casi la mitad (43\%), fueron realizadas entre 8:00 y 10:30 am. En contraste, la actividad en los últimos dos períodos fue bastante baja, sumando apenas un $17 \%$ de las visitas del día.

Tiempo de visita: El tiempo medio de visita fue similar para el polen y néctar en términos generales. Así, para la recolección de polen las abejas usaron $6.5 \pm 3.8 \mathrm{~s}$ y para la de néctar $5.8 \pm 4.1 \mathrm{~s}$.

Por otro lado, observamos que el tiempo de recolección de las abejas estuvo fuertemente relacionado al sexo floral. En las flores femeninas, el tiempo medio de recolección fue siempre superior (cercano a $8.4 \pm 4.4 \mathrm{~s}$ ) al empleado en las flores masculinas (cerca de $4.0 \pm 1.5 \mathrm{~s}$ ). Este mayor tiempo de "manipulación" quizás es reflejo de la mayor complejidad de forma y estructura de las flores femeninas para forzar a las abejas a polinizarlas al acceder a sus nectarios.

\section{DISCUSIÓN}

Como se mencionó, las visitas de abejas a las flores del melón observadas se realizaron mayormente hasta las primeras horas de la tarde. Así, observamos que la mayor parte de la actividad colectora se realizó antes del medio día, totalizando el $65 \%$ de las visitas. Resultados similares fueron obtenidos por McGregor y Todd (1952) y Vaissiere y Froissart (1996) quienes señalan que cerca del $71 \%$ de las visitas fueron realizadas antes de medio día. Además, el patrón de recolección de polen hacia las primeras horas de la mañana puede atribuirse a que éste es un recurso limitado y producido una sola vez por la flor, por lo que su cantidad merma a medida que avanza el día y es demandado por los insectos. El néctar, en cambio, es producido continuamente por la flor, hasta bien avanzada la tarde.

Por otro lado, el número de visitas por néctar fue notablemente mayor al de las visitas por polen (Cuadro 1). Esto puede deberse a que las visitas por polen sólo se realizan hacia las primeras horas de la mañana, entre 7:00 y 11:00 am (4 h en total). Las visitas por polen, en cambio, empezaron desde la mañana (aunque en proporción baja) y continuaron hasta entrada la tarde, cerca de las 5:00 pm.

También hay que destacar que el tiempo medio de visita en las flores femeninas fue significativamente superior (Prueba t Student, $\mathrm{p}<0.0001)$ al observado en las masculinas (Fig. 2). Este patrón es similar al observado por McGregor et al. (1965), quienes reportan un tiempo medio de diez segundos en las visitas de abejas melíferas a las flores femeninas del melón en zonas templadas. Este comportamiento también se presenta en las flores de la sandía, otra cucurbitácea. Según Adlerz (1966) las abejas emplearon mayor tiempo en las flores femeninas $(8.0 \mathrm{~s})$ que en las masculinas (5.7 s).

Así, los resultados obtenidos en la presente investigación nos proporcionan un medio para optimizar la producción en los cultivos de

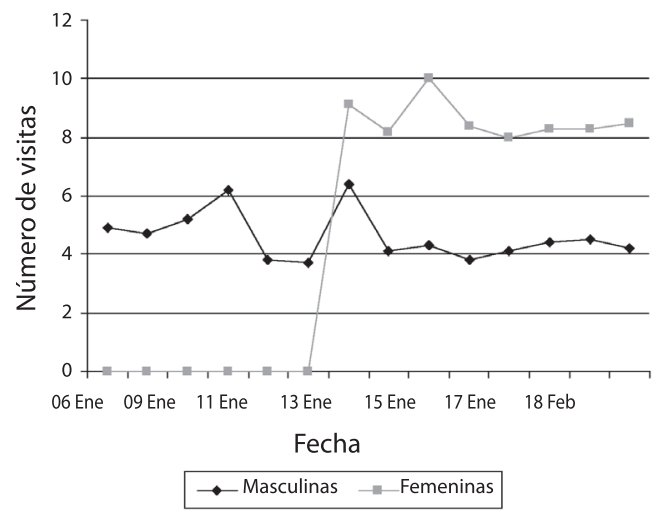

Fig. 2. Tiempo medio de recolección por sexo floral del 6 enero a 19 febrero 2002.

Fig. 2. Mean time of gathering per floral sex from January 6 to February 19, 2002. 
melón. Al mostrarnos las principales horas de actividad de las abejas, se pueden dirigir las fumigaciones o aplicaciones de otros químicos al cultivo a momentos en que no entorpezcan la actividad polinizadora. Además, el número de visitas a cada flor y el tiempo usado por las abejas al polinizar las flores puede ser utilizado, mediante cálculos, para conocer el número de abejas necesarias para polinizar un cultivo de determinado tamaño y cantidad de plantas, y maximizar su rendimiento, tomando en cuenta que el tiempo medio de recolección de recursos, en cada sexo floral, permaneció prácticamente constante conforme evolucionó el cultivo (Fig. 2).

\section{AGRADECIMIENTOS}

El presente artículo es parte de la tesis de Maestría titulada "Abejas Polinizadoras en Cultivos de Melón del distrito de San Lorenzo, Chiriquí", la cual fue asesorada por Cheslavo Korytkouski, de la Universidad de Panamá, y parcialmente financiada por la Empresa Agroexportadora Dos Valles.

\section{RESUMEN}

Se observaron las visitas observadas a flores seleccionadas en un cultivo del Distrito de San Lorenzo, Chiriquí, del 6 de Enero y el 19 de Febrero del 2002, desde las 6:30 am hasta las 4:30 pm, y se anotaron características de las visita, como el tiempo de visita y el tipo de recurso colectado. Las visitas fueron mayormente para la recolección de néctar (casi 3/4). La recolección de polen se concentró hacia las primeras horas de la mañana, cesando definitivamente a las 11:00 am. El tiempo medio de recolección fue similar para ambos recursos, pero fue marcadamente distinto para cada sexo floral. Las visitas a flores femeninas fueron significativamente (Prueba t Student, $\mathrm{p}<0.0001$ ) más largas, con un tiempo medio de duración de $8.4 \pm 4.4 \mathrm{~s}$, mientras que en las flores masculinas, el tiempo medio de visita fue $4.0 \pm 1.5 \mathrm{~s}$. Por último, el tiempo medio de recolección por sexo floral permaneció prácticamente constante conforme evolucionó el cultivo. Nuestros resultados fueron similares a los encontrados en cultivos de zonas templadas, por lo que aparentemente las condiciones tropicales de Panamá no cambian los patrones de visita que presentan las abejas en las flores de melón.

Palabras clave: melón, polinización, visita, recurso, néctar, polen, sexo floral.

\section{REFERENCIAS}

Adlerz,W. 1966. Honey Bee Visit Numbers and Watermelon Pollination. J. Econ. Entomol. 59: 28-30.

Free, J. 1970. Insect Pollination of Crops. Academic, Nueva York, EEUU. 544 p.

Gary, N., P. Whitherell \& J. Marston. 1975. The Distribution of Foraging Honey Bees from Colonies Used for Honeydew Melon Pollination. Env. Ent. 4: 277-280.

Iselin, W., M. Jensen \& H. Spangler. 1974. The Pollination of Melon in Air Inflated Greenhouse by Honey Bees. Env. Ent. 3: 664-666.

McGregor, S. 1976. Insect Pollination of Cultivated Crops. Agriculture Handbook 496. United States Government Printing Office, Washington, DC, EEUU. 411 p.

McGregor, S. \& F. Todd. 1952. Cantaloup Production with Honey Bees. J. Econ. Entomol. 45: 43-47.

McGregor, S., M. Levin \& R. Foster. 1965. Honey Bee Visitors and Fruit Set of Cantaloups. J. Econ. Entomol. 58: 968-970.

Rallo García, J. 1986. La Apicultura Orientada a la Polinización Frutal. Ministerio de Agricultura, Pesca y Alimentación de España 11: 1-27.

Taylor, E. 1955. Cantaloup Production Increased with Honey Bees. J. Econ. Entomol. 48: 327.

Vaissiere, B. \& R. Froissart. 1996. Pollination of Cantaloupe Under Spundbonded Row Cover by Honey Bees (Hymenoptera: Apidae) in West Africa. J. Econ. Entomol. 89: 1213-1222. 